\title{
Cell Adhesion on Dynamic Supramolecular Surfaces Probed by Fluid Force Microscopy- Based Single-Cell Force Spectroscopy
}

Shrikrishnan Sankaran, ${ }^{\dagger, \downarrow, \nabla, \perp}$ Leena Jaatinen, ${ }^{\S, \|, \perp}$ Jenny Brinkmann, ${ }^{\dagger, \dagger}$ Tomaso Zambelli, ${ }^{\|}$Janos Vörös, ${ }^{\oplus \odot}$

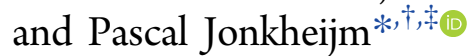

${ }^{\dagger}$ Bioinspired Molecular Engineering Laboratory, MIRA Institute for Biomedical Research and Technical Medicine and ${ }^{\ddagger}$ Molecular Nanofabrication Group, MESA+ Institute for Nanotechnology, University of Twente, P.O. Box 217, 7500 AE Enschede, The Netherlands

${ }^{\S}$ Department of Electronics and Communications Engineering, Tampere University of Technology, BioMediTech, Finn-Medi 1 L 4, Biokatu 6, FI-33520 Tampere, Finland

"Laboratory of Biosensors and Bioelectronics, Institute for Biomedical Engineering, ETH Zurich, CH-8092 Zurich, Switzerland

Supporting Information

ABSTRACT: Biomimetic and stimuli-responsive cell-material interfaces are actively being developed to study and control various cell-dynamics phenomena. Since cells naturally reside in the highly dynamic and complex environment of the extracellular matrix, attempts are being made to replicate these conditions in synthetic biomaterials. Supramolecular chemistry, dealing with noncovalent interactions, has recently provided possibilities to incorporate such dynamicity and responsiveness in various types of architectures. Using a cucurbit $[8]$ uril-based host-guest system, we have successfully established a dynamic and electrochemically responsive interface for the display of the

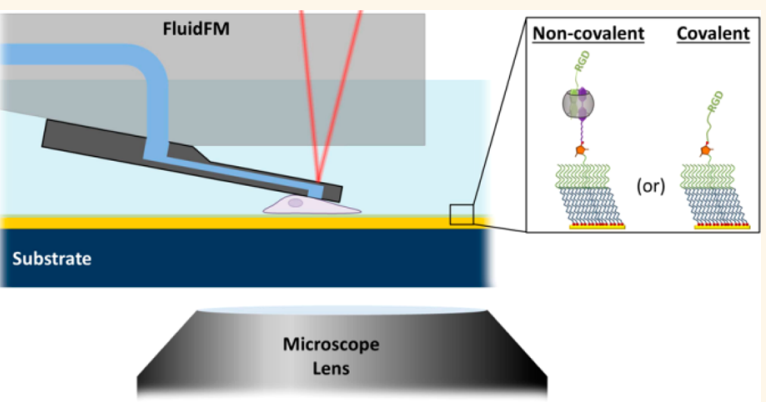
integrin-specific ligand, Arg-Gly-Asp (RGD), to promote cell adhesion. Due to the weak nature of the noncovalent forces by which the components at the interface are held together, we expected that cell adhesion would also be weaker in comparison to traditional interfaces where ligands are usually immobilized by covalent linkages. To assess the stability and limitations of our noncovalent interfaces, we performed single-cell force spectroscopy studies using fluid force microscopy. This technique enabled us to measure rupture forces of multiple cells that were allowed to adhere for several hours on individual substrates. We found that the rupture forces of cells adhered to both the noncovalent and covalent interfaces were nearly identical for up to several hours. We have analyzed and elucidated the reasons behind this result as a combination of factors including the weak rupture force between linear Arg-Gly-Asp and integrin, high surface density of the ligand, and increase in effective concentration of the supramolecular components under spread cells. These characteristics enable the construction of highly dynamic biointerfaces without compromising cell-adhesive properties.

KEYWORDS: supramolecular chemistry, cucurbit[8]urils, self-assembled monolayers, FluidFM, single-cell force spectroscopy

\footnotetext{
Iresis

nteractions between cells and their extracellular matrix (ECM) have been extensively studied over the past couple of decades, yielding specifics on binding partners, motifs, and strengths. These specifics have spurred improved designs of biomaterials aimed to better integrate these materials with cells and tissue. ${ }^{1}$ The identification of the fibronectin-derived tripeptide Arg-Gly-Asp (RGD) as an integral ECM component that mediates cell adhesion via integrins has in particular led to the development of numerous artificial biointerfaces. ${ }^{2-4}$ Consequently, studies have shown that spatial orientation and distribution of this simple peptide influences cell adhesion, spreading, migration, and stem cell differentiation. ${ }^{5-10}$ Stimuli-
}

responsive platforms have additionally allowed us to gain control over the temporal availability of this RGD peptide, resulting in the possibility of eliciting cellular responses. ${ }^{11-14}$

For developing such biomimetic and responsive interfaces, supramolecular systems, dealing with molecular components assembled through noncovalent interactions, have become increasingly attractive. ${ }^{15-17}$ The dynamic nature of the

Received: January 9, 2017

Accepted: March 20, 2017

Published: March 20, 2017 

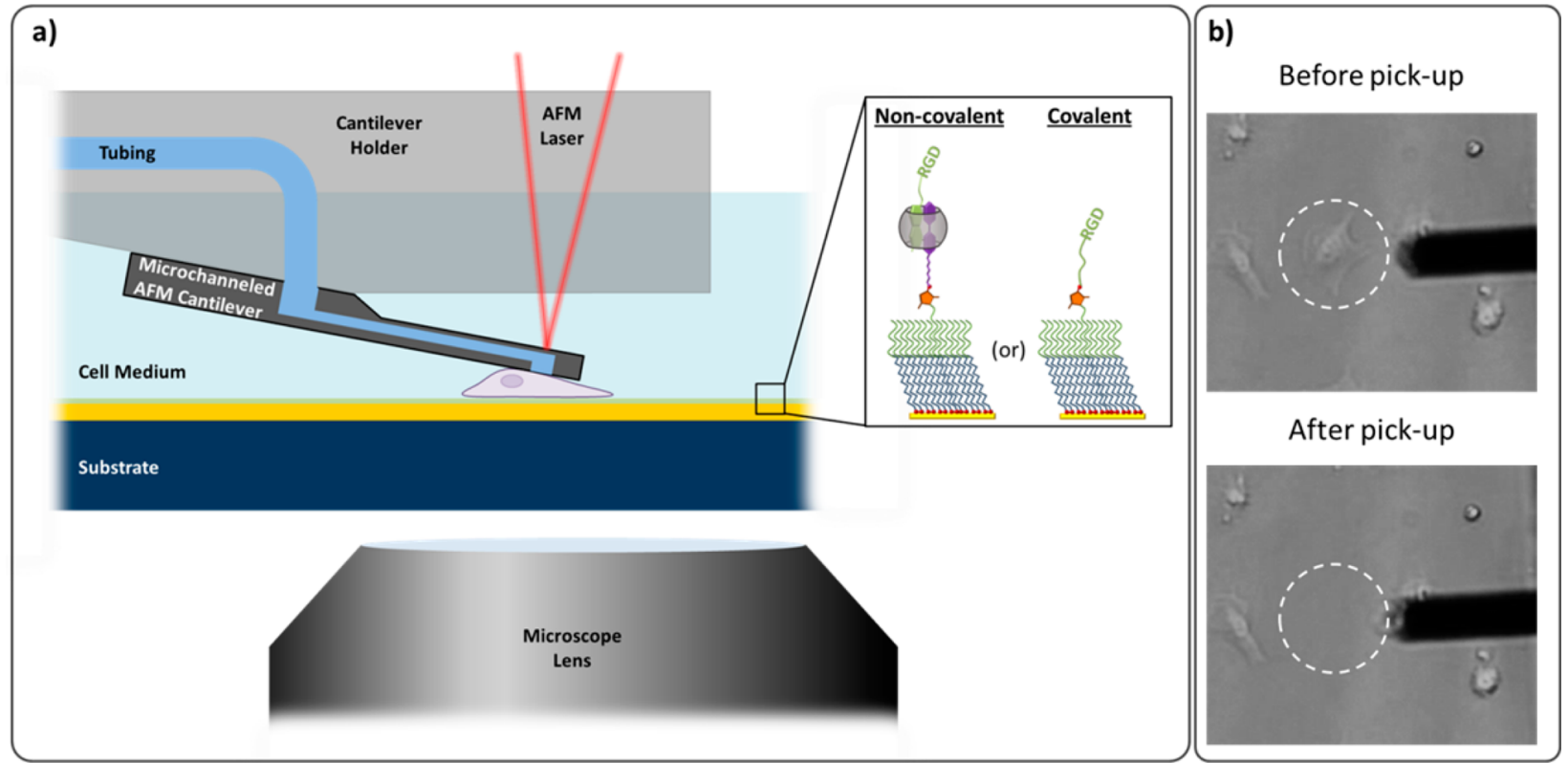

Figure 1. (a) Schematic depicting the FluidFM system for cell-adhesion force spectroscopy, a maleimide-capped SAM onto which MV ${ }^{2+}$, $\mathrm{CB}[8]$, and a naphthol-capped RGD peptide derivative were attached, representing a noncovalent RGD SAM and a maleimide-capped SAM onto which a cysteine-capped RGD peptide derivative was attached representing a covalent RGD SAM. (b) Representative pictures of a cell pick-up experiment. The black bar is the microchannelled cantilever, and the cell that was picked up is shown in the dashed white circle.

individual components in these systems has been suggested to better mimic the ECM, which is known to constantly undergo reorganization. Furthermore, the possibility to introduce stimuli responsiveness by careful selection of individual components allows for manipulation of cells using, for example, $\mathrm{pH}$, electricity, or light. Despite the encouraging progress made using supramolecular systems, a deeper understanding of the constituent interactions is required to make further advances.

Recently, we have shown that self-assembled monolayers (SAMs) of cucurbit[8] uril ( $\mathrm{CB}[8])$ and its associated guests allow the presentation of bioactive ligands in a dynamic and reversible manner. ${ }^{18-22}$ We have used such SAMs to address and manipulate prokaryotic and eukaryotic cells. ${ }^{18-21}$ In particular, SAMs of $\mathrm{CB}[8]$ anchored to the surface using methyl viologen $\left(\mathrm{MV}^{2+}\right)$ as the first electron-poor aromatic guest and a second electron-rich aromatic guest that displays the RGD peptide motif were used for the construction of an electrochemically responsive platform for cell adhesion. ${ }^{18,19}$ Electrochemical reduction of $\mathrm{MV}^{2+}$ to the radical cation form $\left(\mathrm{MV}^{+}\right)$resulted in the disassembly of the ternary complex and enabled the controlled detachment of either the whole cell or a desired subcellular region. Furthermore, the individual hostguest affinities $\left(K_{\mathrm{a}}=10^{5}-10^{6} \mathrm{M}^{-1}\right)^{23,24}$ occur in a range that allows a high level of ligand dynamicity at the surface, especially considering the high rate of association $\left(k_{\mathrm{a}}=9.6 \times 10^{7} \mathrm{M}^{-1}\right.$ $\left.\mathrm{s}^{-1}\right)$ and rapid rate of dissociation $\left(k_{\mathrm{d}}=1200 \mathrm{~s}^{-1}\right)$ for the second guest, naphthol $(\mathrm{Np}){ }^{25}$ Due to these properties of responsiveness and dynamicity, this supramolecular platform could potentially be used as a powerful tool to trigger and study complex cellular phenomena. Despite promising results in preliminary studies, ${ }^{18,19}$ it is possible that cell adhesion does not occur on these noncovalently assembled surfaces to the same extent as it does on more traditional biomaterial surfaces where ligands are immobilized in a covalent manner. ${ }^{26-28}$ For further development of this platform and other such promising supramolecular systems, it is important to gain in-depth understanding of the cellular interactions involved.

With this perspective in mind, we have attempted to gain a better understanding of the forces involved between adhered cells and our supramolecular surfaces using a versatile singlecell force spectroscopy technique called fluid force microscopy (FluidFM). ${ }^{3}$ Previously, single-cell force spectroscopy has been successfully performed by various groups using AFM cantilevers functionalized with cell-adhesive ligands. ${ }^{29-31}$ Such cantilevers have been used to pick up individual cells and bring them in contact with a desired surface. After allowing the cell to adhere for a certain, short period of time, the cantilever would be retracted, and the rupture force between the cell and surface could be determined. Using this technique, rupture forces were determined at scales from single molecules to single cells depending on the time of contact between the cell and surface. ${ }^{29-31}$ However, this versatile technique is immensely time consuming to study whole-cell adhesive forces in a statistically significant manner. Furthermore, since the cell is always held by the AFM cantilever, its adhesive properties toward surfaces might be not representative when compared to when cells adhere freely from solution and stably spread over the course of hours.

In contrast, FluidFM enables the achievement of more relevant force statistics on the scale of entire cells and allows probing cells that have adhered to surfaces in an unhindered manner. ${ }^{32}$ As shown in Figure 1a, FluidFM utilizes an atomic force microscopy (AFM) cantilever with an integrated microchannel. The device functions as an AFM with the possibility to perform aspiration by applying negative pressure and dispensing by applying positive pressure through the microchannel, like a miniscule pipet. For single-cell adhesion force measurements, ${ }^{33}$ a surface with adhered cells is slowly approached with the cantilever until the aperture on the tip of the microchannel makes contact with the target cell. An under pressure is then applied through the microchannel, and the 
cantilever is slowly retracted, resulting in the cell being detached from the surface as shown in Figure $1 \mathrm{~b}$. The entire measurement lasts approximately $10 \mathrm{~min}$ and can be repeated over several hours. Previously, FluidFM has been successfully used for measuring and comparing adhesion forces of HeLa and HEK cells on fibronectin-coated tissue culture plates, ${ }^{33}$ for determining endothelial cell adhesion forces on different charged microstructured substrates ${ }^{34}$ as wells as for studying the changes in adhesion forces of $\mathrm{C} 2 \mathrm{C} 12$ cells due to an applied electric current. ${ }^{35}$

In this study, we performed single-cell force spectroscopy to probe cell adhesive strengths on surfaces with noncovalently immobilized peptide ligands. Using FluidFM, we prove that cells adhere with similar forces to surfaces displaying RGD in a traditional covalent manner and in a dynamic noncovalent manner. We also discuss the possible supramolecular mechanisms behind our results.

\section{RESULTS}

Two types of RGD-presenting surfaces were analyzed by celladhesion force measurements. Both surfaces were made up of a well-packed background mixed monolayer consisting of dimeric tetraethylene glycol alkanethiols in which $1 \%$ contained reactive maleimide groups (Figure 1a). In the case of the covalent surfaces, an RGD peptide with a cysteine residue was conjugated directly to the maleimide. In the case of the noncovalent surfaces, a thiolated $\mathrm{MV}^{2+}$ was conjugated to the maleimides to which $\mathrm{CB}[8]$ was able to bind. An RGDcontaining peptide conjugated with a naphthol unit (Np-RGD) acted as the second guest and allowed RGD to be presented on the surface in a noncovalent way. On both of these surfaces, cell spreading occurred similarly over several hours using mouse myoblast cells ( $\mathrm{C} 2 \mathrm{C} 12)$, as indicated by their cell areas in Figure 2a. A $\mathrm{MV}^{2+}$-conjugated surface (without both $\mathrm{CB}[8]$ and $\mathrm{Np}-\mathrm{RGD}$ ) and a $\mathrm{MV}^{2+}$ with $\mathrm{CB}[8]$ were used as the negative control surfaces on which cells adhered less and remained rounded for several hours, indicating that the adhesion of cells was indeed mediated by specific interactions to RGD (Figure 2a, Supporting Information Figure S1 and S2). Cells adhering to both covalent and noncovalent surfaces had well-formed focal adhesions and actin networks during the course of several hours (Figure $2 b$ ). These results verified that cell adhesion and spreading occurred in a similar manner on both covalent and noncovalent surfaces.

Cell-adhesion force measurements were then performed on three types of surfaces using the FluidFM device. A typical force spectroscopy curve from such cell pick-up experiments is presented in Figure 3a. The initial drop in force represents the bending of the cantilever that is being retracted after being brought in contact with a cell. The maximum adhesion force is the point where the force to bend the cantilever equals the celladhesion force. Beyond this point, the cell detaches from the surface, and the cantilever returns to its original shape, represented by the force curve returning to its initial value. The total distance required to pull a cell off the surface is measured from the initial point where the cantilever bending begins to when it reaches its original shape again. The area of the shaded region in the force-distance curve represents the work done by the cantilever to detach a cell from the surface and corresponds to the binding energy of a cell with the surface. Representative force-distance curves for the three types of substrates used are presented in the Supporting Information (Figure S3).
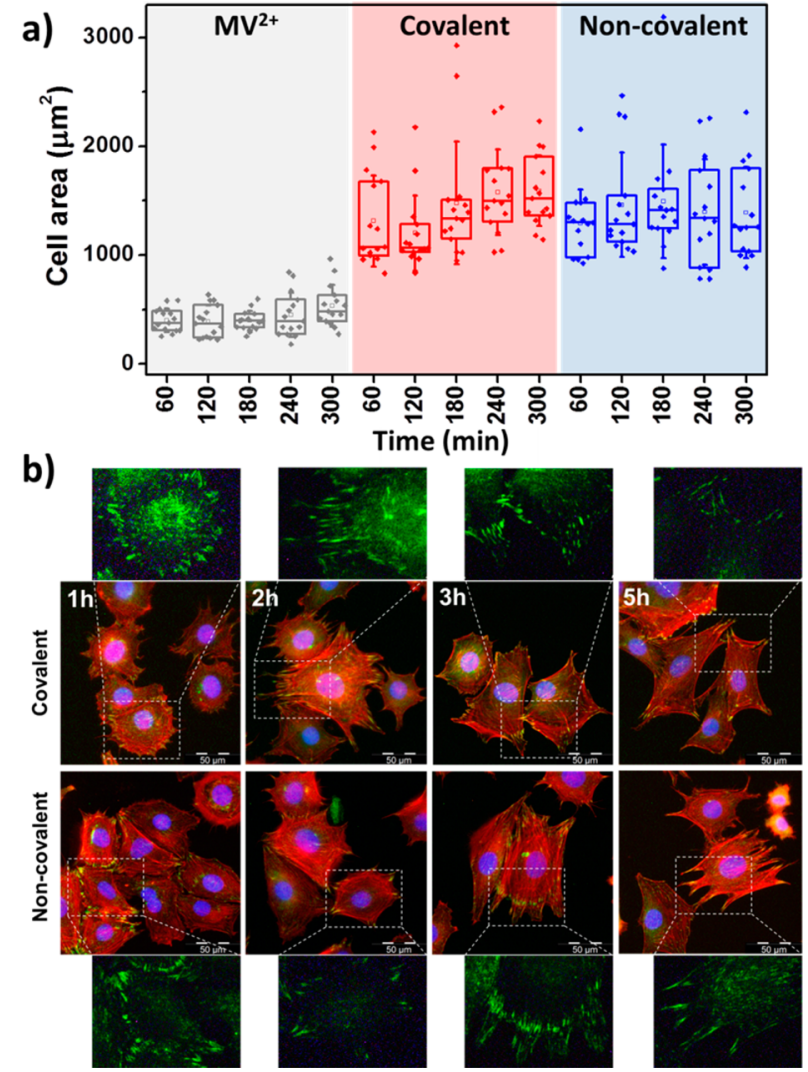

Figure 2. (a) Plot of cell areas on $\mathrm{MV}^{2+}$, covalent, and noncovalent surfaces over a period of $5 \mathrm{~h}$ after cell seeding. The top and bottom of the boxes correspond to the first and third quartiles, the line in the middle corresponds to the median, the hollow squares represent the mean, and the whiskers represent the standard deviation (STD) of the data sets. Mean \pm STD area values for all the cells measured on $\mathrm{MV}^{2+}$ surfaces $=419 \pm 168 \mu \mathrm{m}^{2}$, covalent surface $=1353 \pm 519 \mu \mathrm{m}^{2}$, and noncovalent surfaces $=1330 \pm 523$ $\mu \mathrm{m}^{2}$. (b) Fluorescence overlaid images of $\mathrm{C} 2 \mathrm{C} 12$ cells on the covalent and noncovalent surfaces, stained to visualize actin (red), focal adhesion protein vinculin (green), and cell nuclei (blue). Magnified images of the vinculin staining have been provided for better visibility of the focal adhesion. Time points indicated on the top left of the images correspond to number of hours after cell seeding.

Cells were allowed to adhere and spread on the covalent and noncovalent surfaces for an hour after which the force-distance curves were measured for a time period of up to $4 \mathrm{~h}$. Additionally, gold surfaces coated with serum proteins were used as the standard for comparing the values obtained from the two types of surfaces. The mean \pm STD cell area on this surface was determined to be $919 \pm 314 \mu \mathrm{m}^{2}$. In total, 10 cells were successfully picked up from the serum-coated surfaces, 40 cells from the covalent surfaces, and 49 cells from the noncovalent surfaces. This represents approximately 50\% of all attempted pick ups at an average rate of 4 attempts per hour. Two substrates were used for serum-coated surfaces and seven each for the covalent and noncovalent surfaces. The rounded cells on the negative control surfaces did not display any measurable detachment force and were not included in the analysis. From Figure $3 b-d$, it can be seen that the maximum force, the detachment distance, and the total work required to detach the cells from both covalent and noncovalent surfaces were very similar and comparable to the serum-coated surfaces. 

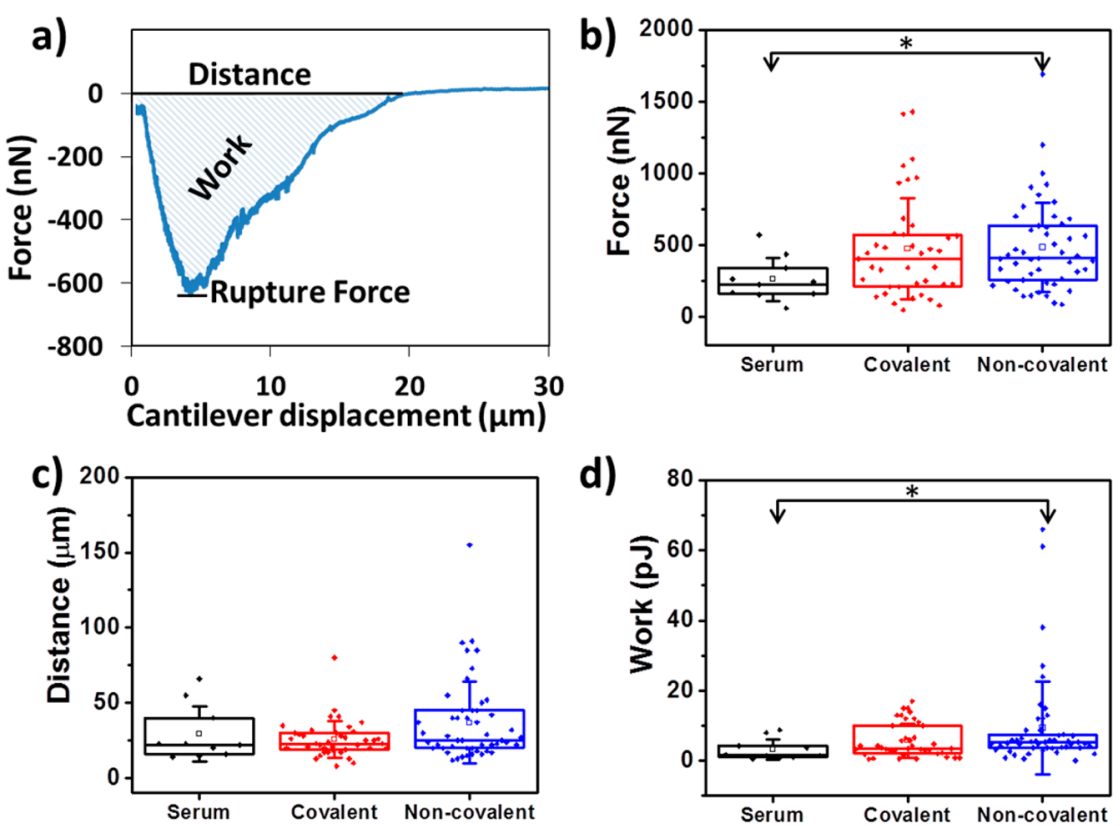

Figure 3. (a) Representative force-distance curve beginning right after the contact between the cell and the cantilever was established. Box plots of the (b) maximum adhesion force, (c) detachment distance, and (d) total work done in the cell pick-up experiments from gold surfaces coated with serum proteins $(n=10$ cells), covalently bound RGD $(n=40$ cells), and noncovalently bound RGD $(n=49$ cells). The top and bottom of the boxes correspond to the first and third quartiles, the line in the middle corresponds to the median, the hollow squares represent the mean, and the whiskers represent the standard deviation (STD) of the data sets. Statistical significance between unpaired data sets determined by the Mann-Whitney U-test have been denoted by a for $p<0.05$.
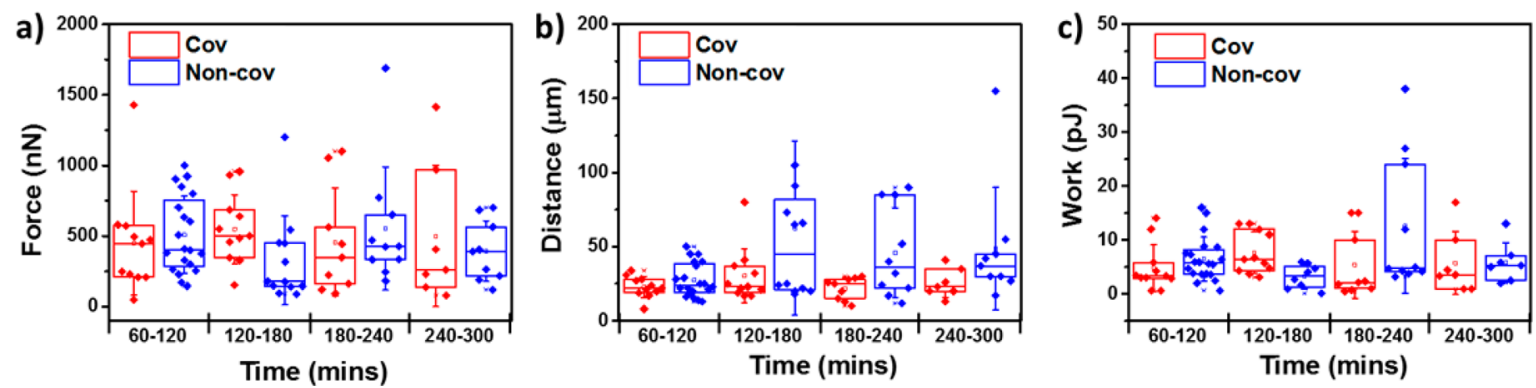

Figure 4. Box plots of the (a) maximum adhesion force, (b) distance, and (c) work done in the cell pick-up experiments from gold surfaces with covalently and noncovalently bound RGD binned over successive time periods. The top and bottom of the boxes correspond to the first and third quartiles, the line in the middle corresponds to the median, and the whiskers represent the standard deviation of the data sets.

The median force values for cell detachment in the covalent $(425 \mathrm{nN})$ and noncovalent $(409 \mathrm{nN})$ samples were higher than that of the serum-coated surface $(236 \mathrm{nN})$, indicating that cells adhered well to these surfaces. Since the data sets follow Gaussian distribution with outliers, the nonparametric MannWhitney U-test was applied, and it was determined that significant differences did not exist between the covalent and noncovalent surfaces for any of the three parameters. However, statistical significance was observed between the serum-coated and noncovalent surfaces in terms of force and work values for cell detachment. The results suggest that, even though the components in the noncovalent surfaces were held together by relatively weak forces, cell adhesion strength was similar to covalent surfaces and better than serum-coated surfaces. From the mean rupture force and cell surface area values, the rupture force density was calculated to be approximately $0.35 \mathrm{nN} / \mu \mathrm{m}^{2}$ for the covalent system and $0.36 \mathrm{nN} / \mu \mathrm{m}^{2}$ for the noncovalent system. These values indicate that both types of surfaces very likely produce a similar number of RGD-integrin interactions per unit area and are in accordance with values obtained in previous studies using covalently immobilized RGD surfaces. $^{29,36}$ Furthermore, there were no statistically significant differences in the cell detachment distances between the three experimental groups in accordance with the fact that the cell areas on all three surfaces were similar.

Since the supramolecular ternary complex on the noncovalent surface can completely dissociate from the surface within an hour, cell adhesion toward this surface may weaken over time. To address this, the force, distance, and work values were binned over successive $1 \mathrm{~h}$ time periods from 1 to $5 \mathrm{~h}$ after cell seeding (Figure 4). It can be seen that cells on covalent surfaces do not show any significant change in celladhesion parameters over time, as expected. The cells adhering to noncovalent surfaces also clearly display no significant decline in all three parameters, indicating that the noncovalently bound RGD ligand does not entirely dissociate from the surface in this time period. 


\section{DISCUSSION}

Traditionally, bioactive ligands are immobilized onto biomaterials through stable covalent linkages, ensuring that the ligands are always available for cells to interact with them. However, in nature, most ligands are presented in a constantly changing and dynamic environment. Mimicking such an environment has proven to be a considerable challenge for which supramolecular chemistry has recently provided innovative solutions. ${ }^{15}$ Using host-guest systems, ligands can be presented in a dynamic and responsive manner, but the forces by which they are held together are much weaker in comparison to covalent bonds. For instance, covalent bonds have rupture forces in the order of $\mathrm{nNs}^{37}$ whereas a typical host-guest complex such as $\beta$-cyclodextrin $(\beta$-CD)-adamantane (Ad) $\left(K_{\mathrm{a}}=5 \times 10^{4} \mathrm{M}^{-1}\right)$ has a rupture force of $100 \mathrm{pN}^{38}$ Based on these values, it is natural to expect that such hostguest complexes would perform poorly when addressing cell behavior such as adhesion to surfaces. In spite of this, we have observed that cell adhesion, spreading, and contractility on our host-guest complex-based noncovalent surfaces are very similar to what is seen on covalent surfaces (Figure $2 b$ ). In this study, we discovered that cell-adhesive forces on our noncovalent surfaces are comparable to that of covalent surfaces. To understand this, we took a closer look into the binding parameters of these systems. In the covalent system, RGD is rigidly bound to the surface, so the weakest link between the cell and the surface is the noncovalent interaction between the RGD peptide and the integrins residing in the cell membrane. The measured rupture force most likely corresponds to the breaking of these interactions. The rupture force in the noncovalent system being similar to that of the covalent system indicates that in both systems, the RGD-integrin complex is probably the weakest link. The association and dissociation rate constants between $\mathrm{Np}$ and the $\mathrm{CB}[8]-\mathrm{MV}^{2+}$ complex $\left(k_{\mathrm{a}}=9.6 \times 10^{7} \mathrm{M}^{-1} \mathrm{~s}^{-1}, k_{\mathrm{d}}=1200 \mathrm{~s}^{-1}\right)^{25}$ are similar to that of the $\beta$-CD-Ad complex mentioned above $\left(k_{\mathrm{a}}=10^{8} \mathrm{M}^{-1}\right.$ $\left.\mathrm{s}^{-1}, k_{\mathrm{d}}=2000 \mathrm{~s}^{-1}\right) .^{38}$ So, it is fair to assume that the rupture force of the ternary complex could be comparable to the 100 $\mathrm{pN}$ of the $\beta$-CD-Ad complex based on a theory developed by Evans et al., ${ }^{39}$ which predicts that the rupture force is inversely proportional to $\ln \left(k_{\mathrm{d}}\right)$. In addition, force spectroscopy studies on individual RGD-integrin complexes have shown that the force required to disassemble this complex is approximately 40 $\mathrm{pN}^{29,40}$ The RGD-integrin rupture force being less than half compared to that of the $\mathrm{Np}-\left(\mathrm{CB}[8]-\mathrm{MV}^{2+}\right)$ complex supports our weakest link hypothesis.

Another observation is that the rupture force of cells adhered on our noncovalent surfaces does not diminish over several hours. Dissociation of $\mathrm{Np}$ and $\mathrm{CB}[8]$ from the surface is expected to occur over time in the cell medium since the equilibrium would be shifted to favor the dissociated state. In previous studies using QCM and SPR, we determined that such dissociation occurs in neutrally buffered solutions within tens of minutes. ${ }^{19}$ To understand how cells remain adhered to our noncovalent surfaces over several hours, we considered the ligand densities at the surface. Mooney and co-workers have shown that $\mathrm{C} 2 \mathrm{C} 12$ cells adhere and spread well on surfaces with RGD ligand densities as low as $1 \mathrm{fmol} / \mathrm{cm}^{2}$ (corresponding to 6 ligands per $\left.\mu \mathrm{m}^{2}\right) .{ }^{36}$ In another study, Heilshorn and coworkers varied the RGD density on their surfaces from 16 to $160 \mathrm{pmol} / \mathrm{cm}^{2}$ and concluded that even their lowest density was sufficient to saturate the integrin receptors on the surface of
$\mathrm{C} 2 \mathrm{C} 12$ cells. $^{41}$ On the $1 \%$ mol maleimide SAM that was used, based on the dimensions of $\mathrm{CB}[8]$ (outer diameter $=1.75$ $\mathrm{nm})^{42}$ and assuming hexagonal packing, a maximum surface density of $\mathrm{CB}[8]$ and $\mathrm{Np}-\mathrm{RGD}$ can be $c a .61 \mathrm{pmol} / \mathrm{cm}^{2}$. This indicates that the RGD ligands density on our noncovalent surfaces is very likely a few orders of magnitude higher than what is sufficient for cell adhesion and spreading even if considerable dissociation of the ligand occurs. Furthermore, once seeded, cells settle on the surface and adhere within a matter of minutes. After adhesion and spreading, the cell membrane is typically separated from the underlying surface by the cellular glycocalyx, which has been determined to span ca. $10-20 \mathrm{~nm} .{ }^{43}$ Such a cell, spread on the noncovalent surfaces, would prevent rapid dissociation of the supramolecular components by limiting the free volume available to them and consequently increasing their effective concentration. Under the cell membrane, assuming an average distance from the surface to be $20 \mathrm{~nm}$, the noncovalently interacting molecules would experience a local concentration of $\sim 30$ $\mathrm{mM}$. This is 3 orders of magnitude higher than the dissociation constant of the $\mathrm{Np}-\left(\mathrm{CB}[8]-\mathrm{MV}^{2+}\right)$ complex $\left(K_{\mathrm{d}}=12.5 \mu \mathrm{M}\right){ }^{19}$ Thus, within the time period in which cell adhesion and spreading occurs, there would still exist a sufficient density of ligands at the surface to ensure that the equilibrium favors the associated state of the supramolecular ternary complex. All of these factors combined would be able to ensure stable cell adhesion on noncovalent surfaces over the time span that was probed.

\section{CONCLUSIONS}

In this study, we have shown that the actin filaments, focal adhesions, adhesion forces, and cell contractility between cells adhering to the covalent and noncovalent surfaces are comparable using fluorescence microscopy and FluidFMbased single-cell force spectroscopy. Although the bioactive ligands are held together through relatively weaker forces in the noncovalent surfaces compared to the covalent surfaces, the total cell adhesive forces on both surfaces were found to be very similar. We postulated that such a surprising result is due to a combination of factors including the weak rupture force between linear RGD and integrin, high surface density of the ligand, and increase in effective concentration of the supramolecular components under spread cells. The platform presented is dynamic, responsive, and can be modified in a highly versatile manner. The ligand's affinity to the surface can be modified by using other or multivalent guest molecules. RGD's affinity toward integrins can be modified by either using cyclic RGD or introducing different adjacent amino acids. The combination of noncovalent surfaces and FluidFM represents a powerful system for gaining further insights into the intricate molecular mechanisms of cell-surface interactions. Further investigations are being conducted by modulating the bioactive ligand's binding strength, valency, and stimuli responsiveness to understand the effects of these parameters on cell contractility and migration.

\section{MATERIALS AND METHODS}

Materials. Cucurbit[8]uril, cucurbit[7]uril, $\beta$-trypsin from bovine pancreas, and methyl viologen were purchased from Sigma-Aldrich. The disulfides (bis-1-(11-\{tetraethylene glycol\}-undecyl) disulfide $\left(\mathrm{EG}_{4} \mathrm{C}_{11} \mathrm{~S}\right)_{2}$ and $\mathrm{N}$-\{2-(2,5-dioxo-2,5-dihydro-pyrrol-1-yl)-ethyl $\}$-[2[11-(11-(tetraethylene glycol)-undecyldisulfanyl)-undecyloxy]-hexaethylene glycol-acetamide]) Mal-EG $\mathrm{C}_{6} \mathrm{CSC}_{11} \mathrm{EG}_{4}$ ) for SAM prepara- 
tion were purchased from ProChimia. Alkyl thiol terminated $\mathrm{MV}^{2+}$ $\left(\mathrm{MV}^{2+}-\mathrm{SH}\right)$ was synthesized as previously reported. ${ }^{44}$ NpGGRGDSG (Np-RGD) was synthesized using an automatic solid-phase synthetic robot (Syro II, Multisyntech) following standard Fmoc procedures on RinkAmide resin. GGCGGRGDS (C-RGD) was synthesized with a MultiPep RSi, Intavis Bioanalytical Instruments using standard solidphase peptide synthesis protocols on a Wang resin. Purification was done by reversed-phase high-performance liquid chromatography (HPLC (Waters)), followed by analysis with analytical HPLC and mass spectrometry. Because of the poor solubility of $\mathrm{CB}[8]$ in water and its hygroscopic nature, the apparent molecular weight of the commercial powder and its actual concentration in aqueous solutions were determined for each batch using a simple and highly reproducible method described previously. ${ }^{45} \mathrm{CB}[8]$ was dissolved in Milli-Q water by sonication at $80^{\circ} \mathrm{C}$ for $2 \mathrm{~h}$.

Substrates and Chamber. As a substrate for the cell adhesion, we used a $4.9 \times 4.9 \mathrm{~cm}$ glass substrates coated with a $20 \mathrm{~nm}$-thick gold layer. Appropriate monolayers were assembled on these substrates as already described. ${ }^{18,20}$ The cell chamber consisted of a poly(methyl methacrylate) base and a polytetrafluoroethylene housing. The chamber and the substrate together were cleaned by incubating with Tergazyme (Alconox, USA) for $20 \mathrm{~min}$, followed by thorough rinsing with Milli-Q. The chamber was sterilized in $70 \%$ ethanol for at least 20 min and allowed to dry under sterile conditions before mounting the substrate.

Preparation of SAMs on Gold Substrate. Gold substrates were first washed with piranha solution $\left(\mathrm{H}_{2} \mathrm{SO}_{4}+30 \% \mathrm{H}_{2} \mathrm{O}_{2}\right.$, v/v 3/1), copious amounts of Milli- $\mathrm{Q}$ water, and finally with ethanol. Substrates were then immersed overnight in a $1 \mathrm{mM}$ ethanolic solution of $\left(\mathrm{EG}_{4} \mathrm{C}_{11} \mathrm{~S}\right)_{2}$ and $\mathrm{Mal}-\mathrm{EG}_{6} \mathrm{C}_{11} \mathrm{SSC}_{11} \mathrm{EG}_{4}$ at a molar ratio of 99:1 at room temperature in the dark. The substrates were then cleaned thoroughly with ethanol, Milli-Q water, and dried with a stream of $\mathrm{N}_{2}$ gas. They were then immediately incubated with $1 \mathrm{mM} \mathrm{MV}^{2+}-\mathrm{SH}$ in pH $6.850 \mathrm{mM}$ phosphate buffer for $1 \mathrm{~h}$. The substrates were then washed thoroughly with Milli- $\mathrm{Q}$ water, dried with $\mathrm{N}_{2}$ gas, and used for further conjugation of either $\mathrm{MV}^{2+}-\mathrm{SH}$ or C-RGD.

The serum-coated surfaces were prepared by incubating clean gold substrates in DMEM/F-12 medium supplemented with $10 \%$ fetal bovine serum (FBS) for a half hour to allow adhesion of serum proteins onto gold. These substrates were then directly used for culturing cells.

FluidFM. A custom built FluidFM platform, called Skeleton, ${ }^{46}$ was used together with a tipless FluidFM cantilever (Cytosurge AG, Zürich, Switzerland) with an aperture of $8 \mu \mathrm{m}$. The cantilever spring constant $(k)$ was calibrated using the Sader method ${ }^{48}$ and was found to be between 1.7 and $2.3 \mathrm{~N} / \mathrm{m}$. The bending of the cantilever was measured by optical beam deflection (OBD), ${ }^{47}$ and the position of the beam on a photodetector was measured in volts $(V)$. Before each experiment, the deflection sensitivity $(S[\mathrm{~V} / \mathrm{nm}])$ of the cantilever was measured by allowing the cantilever to bend on a cell-free spot on the substrate and relax again during which changes in the photodetector voltage was monitored. The force was derived from

$$
F=V S k
$$

The Skeleton was operated on a Zeiss AXIOVERT 200 inverted microscope (Carl Zeiss AG, Jena, Germany). The over- and underpressure in the FluidFM cantilever were established with a pressure controller (Cytosurge) in a range from $-800 \mathrm{mbar}$ to +1000 mbar with $1 \mathrm{mbar}$ resolution and a settling time of $200 \mathrm{~ms}$.

Mouse myoblast $\mathrm{C} 2 \mathrm{C} 12$ cells (American Type Cell Collection) were used in all the experiments. Cells were cultured in DMEM/F-12 supplemented with 10\% FBS and 1\% antibiotic-antimycotic (all from Thermo Fisher Scientific AG, Switzerland). Approximately 100,000 cells were seeded on the substrates in $4 \mathrm{~mL}$ of serum-free DMEM (Thermo Fisher Scientific AG, Switzerland) and allowed to adhere and spread for $1 \mathrm{~h}$ before the force-distance curves were measured. DMEM with serum was used for cells seeded on serum-coated surfaces.

Prior to the force measurements, the cantilever was filled with the Milli-Q water by applying an overpressure. Individual pick-ups were performed by approaching a cell with the cantilever at $1 \mu \mathrm{m} / \mathrm{s}$, maintaining +20 mbar overpressure, and stopping for $10 \mathrm{~s}$ when a $5 \%$ deflection in the photodetector voltage was detected due to the bending of the cantilever when making contact with the cell. At this point, the cantilever was kept static for $10 \mathrm{~s}$, enough time to apply an underpressure of -800 mbar after which it was retracted with a velocity of $1 \mu \mathrm{m} / \mathrm{s}$. As the cantilever is retracted, it bends downward since the cell is still adhered to the surface with a certain force. Once the force required to bend the cantilever exceeds the maximum celladhesion force, a rupture event occurs, causing the cell to be detached from the surface and the cantilever to return to its original shape.

All experiments were performed at $37{ }^{\circ} \mathrm{C}$ in a humidified $5 \% \mathrm{CO}_{2}$ atmosphere.

Between every adhesion force measurement, the measurement chamber was replaced with the cleaning chambers with sodium hypochlorite and Milli-Q water. The cantilever was cleaned by first dipping it in 5\% sodium hypochlorite and then thrice in pure Milli-Q water. This prevented the cantilever from accumulating extracellular matrix and allowed to use it repeatedly over the course of many days. Including the cleaning procedure, up to 6 cells could be measured per hour. Between the experiments, the cantilevers were stored in a MilliQ water supplemented with $2 \%$ of antibiotic-antimycotic (Thermo Fisher Scientific AG, Switzerland)

Immunocytochemistry. Cells grown on substrates were fixed in $4 \%$ paraformaldehyde for $10 \mathrm{~min}$, rinsed $3 \times$ in PBS, permeabilized in $0.5 \%$ Triton X-100 (TX) for $10 \mathrm{~min}$, and blocked with $0.1 \% \mathrm{TX}$ and $5 \%$ bovine serum albumin (PBST) for $30 \mathrm{~min}$ at room temperature (RT). Incubation of 1:100 monoclonal vinculin-FITC and 1:100 phalloidin 568 was done for $1 \mathrm{~h}$ at RT in PBST, followed by washing $2 \times$ in PBST and incubation 1:1000 with DAPI for $10 \mathrm{~min}$ at RT in PBS. Cells were rinsed twice in PBS and imaged using an inverted fluorescent microscope with corresponding excitation and emission filters (Olympus, 1X71, Melville NY, USA).

\section{ASSOCIATED CONTENT}

\section{Supporting Information}

The Supporting Information is available free of charge on the ACS Publications website at DOI: 10.1021/acsnano.7b00161.

Figures S1 and S2: Bright-field images of cell adhesion and spreading on methyl viologen, methyl viologen/ $\mathrm{CB}[8]$, covalent, and noncovalent RGD surfaces; and Figure S3: Representative force-distance curves for the different substrates (PDF)

\section{AUTHOR INFORMATION}

\section{Corresponding Author}

*E-mail: p.jonkheijm@utwente.nl.

ORCID

Janos Vörös: 0000-0001-6054-6230

Pascal Jonkheijm: 0000-0001-6271-0049

\section{Present Address}

${ }^{\nabla}$ Dynamic Biomaterials, INM - Leibniz-Institut für Neue Materialien gGmbH, Campus D2 2, 66123 Saarbrücken, Germany

\section{Author Contributions}

${ }^{\perp}$ These authors contributed equally. S.S. and L.J. performed FluidFM measurements and analysis. Surfaces were fabricated by S.S. and J.B. Fluorescence microscopy imaging was performed by J.B.

\section{Notes}

The authors declare no competing financial interest. 


\section{ACKNOWLEDGMENTS}

We thank Dr. Emanuela Cavatorta for providing us with thiolated methylviologen $\left(\mathrm{MV}^{2+}-\mathrm{SH}\right)$. The European Research Council through Starters Grant 259183 and the Swiss NCCR Molecular Systems Engineering are acknowledged for funding. This research forms part of the Project P4.02 Superdices of the research program of the BioMedical Materials institute, cofunded by the Dutch Ministry of Economic Affairs, Agriculture and Innovation.

\section{REFERENCES}

(1) Mouw, J. K.; Ou, G.; Weaver, V. M. Extracellular Matrix Assembly: A Multiscale Deconstruction. Nat. Rev. Mol. Cell Biol. 2014, $15,771-785$.

(2) Ruoslahti, E.; et al. RGD and Other Recognition Sequences for Integrins. Annu. Rev. Cell Dev. Biol. 1996, 12, 697-715.

(3) Dörig, P.; Stiefel, P.; Behr, P.; Sarajlic, E.; Bijl, D.; Gabi, M.; Vörös, J.; Vorholt, J. A.; Zambelli, T. Force-Controlled Spatial Manipulation of Viable Mammalian Cells and Micro-Organisms by Means of FluidFM Technology. Appl. Phys. Lett. 2010, 97, 023701.

(4) Hersel, U.; Dahmen, C.; Kessler, H. RGD Modified Polymers: Biomaterials for Stimulated Cell Adhesion and Beyond. Biomaterials 2003, 24, 4385-4415.

(5) Huang, J.; Gräter, S. V.; Corbellini, F.; Rinck-Jahnke, S.; Bock, E.; Kemkemer, R.; Kessler, H.; Ding, J.; Spatz, J. P. Impact of Order and Disorder in RGD Nanopatterns on Cell Adhesion. Nano Lett. 2009, 9, $1111-1116$

(6) Pallarola, D.; Bochen, A.; Boehm, H.; Rechenmacher, F.; Sobahi, T. R.; Spatz, J. P.; Kessler, H. Interface Immobilization Chemistry of cRGD-Based Peptides Regulates Integrin Mediated Cell Adhesion. Adv. Funct. Mater. 2014, 24, 943-956.

(7) Schwab, E. H.; Halbig, M.; Glenske, K.; Wagner, A.-S.; Wenisch, S.; Cavalcanti-Adam, E. A. Distinct Effects of RGD-Glycoproteins on Integrin-Mediated Adhesion and Osteogenic Differentiation of Human Mesenchymal Stem Cells. Int. J. Med. Sci. 2013, 10, 1846-1859.

(8) Fittkau, M. H.; Zilla, P.; Bezuidenhout, D.; Lutolf, M. P.; Human, P.; Hubbell, J. A.; Davies, N. The Selective Modulation of Endothelial Cell Mobility on RGD Peptide Containing Surfaces by YIGSR Peptides. Biomaterials 2005, 26, 167-174.

(9) Rahmany, M. B.; Van Dyke, M. Biomimetic Approaches to Modulate Cellular Adhesion in Biomaterials: A Review. Acta Biomater. 2013, 9, 5431-5437.

(10) Yang, F.; Williams, C. G.; Wang, D.; Lee, H.; Manson, P. N.; Elisseeff, J. The Effect of Incorporating RGD Adhesive Peptide in Polyethylene Glycol Diacrylate Hydrogel on Osteogenesis of Bone Marrow Stromal Cells. Biomaterials 2005, 26, 5991-5998.

(11) Petersen, S.; Alonso, J. M.; Specht, A.; Duodu, P.; Goeldner, M.; del Campo, A. Phototriggering of Cell Adhesion by Caged Cyclic RGD Peptides. Angew. Chem., Int. Ed. 2008, 47, 3192-3195.

(12) Lee, T. T.; García, J. R.; Paez, J. I.; Singh, A.; Phelps, E. A.; Weis, S.; Shafiq, Z.; Shekaran, A.; del Campo, A.; García, A. J. LightTriggered in Vivo Activation of Adhesive Peptides Regulates Cell Adhesion, Inflammation and Vascularization of Biomaterials. Nat. Mater. 2015, 14, 352-360.

(13) Iturri, J.; García-Fernández, L.; Reuning, U.; García, A. J.; del Campo, A.; Salierno, M. J. Synchronized Cell Attachment Triggered by Photo-Activatable Adhesive Ligands Allows QCM-Based Detection of Early Integrin Binding. Sci. Rep. 2015, 5, 9533.

(14) Yeo, W.-S.; Hodneland, C. D.; Mrksich, M. Electroactive Monolayer Substrates That Selectively Release Adherent Cells. ChemBioChem 2001, 2, 590-593.

(15) Brinkmann, J.; Cavatorta, E.; Sankaran, S.; Schmidt, B.; van Weerd, J.; Jonkheijm, P. About Supramolecular Systems for Dynamically Probing Cells. Chem. Soc. Rev. 2014, 43, 4449-4469.

(16) Uhlenheuer, D. A.; Petkau, K.; Brunsveld, L. Combining Supramolecular Chemistry with Biology. Chem. Soc. Rev. 2010, 39, 2817-2826.
(17) Boekhoven, J.; Stupp, S. I. 25th Anniversary Article: Supramolecular Materials for Regenerative Medicine. Adv. Mater. 2014, 26, 1642-1659.

(18) An, Q.; Brinkmann, J.; Huskens, J.; Krabbenborg, S.; de Boer, J.; Jonkheijm, P. A Supramolecular System for the Electrochemically Controlled Release of Cells. Angew. Chem., Int. Ed. 2012, 51, 1223312237.

(19) Brinkmann, J.; Sankaran, S.; Rinnen, S.; Arlinghaus, F. H.; De Boer, J.; Jonkheijm, P. Dynamic Bioactive Surface for Integrin Mediated Cell Dynamics; Nat. Commun. submitted.

(20) Sankaran, S.; Kiren, M. C.; Jonkheijm, P. Incorporating Bacteria as a Living Component in Supramolecular Self-Assembled Monolayers through Dynamic Nanoscale Interactions. ACS Nano 2015, 9, 35793586.

(21) Cavatorta, E.; Verheijden, M. L.; van Roosmalen, W.; Voskuhl, J.; Huskens, J.; Jonkheijm, P. Functionalizing the Glycocalyx of Living Cells with Supramolecular Guest Ligands for Cucurbit[8]urilMediated Assembly. Chem. Commun. 2016, 52, 7146-7149.

(22) Sankaran, S.; de Ruiter, M.; Cornelissen, J. J. L. M.; Jonkheijm, P. Supramolecular Surface Immobilization of Knottin Derivatives for Dynamic Display of High Affinity Binders. Bioconjugate Chem. 2015, 26, 1972-1980.

(23) Bush, M. E.; Bouley, N. D.; Urbach, A. R. Charge-Mediated Recognition of N-Terminal Tryptophan in Aqueous Solution by a Synthetic Host. J. Am. Chem. Soc. 2005, 127, 14511-14517.

(24) Appel, E. A.; Dyson, J.; del Barrio, J.; Walsh, Z.; Scherman, O. A. Formation of Single-Chain Polymer Nanoparticles in Water through Host-guest Interactions. Angew. Chem., Int. Ed. 2012, 51, 4185-4189.

(25) Appel, E. A.; Biedermann, F.; Rauwald, U.; Jones, S. T.; Zayed, J. M.; Scherman, O. A. Supramolecular Cross-Linked Networks via Host-guest Complexation with cucurbit[8]uril. J. Am. Chem. Soc. 2010, 132, 14251-14260.

(26) Massia, S. P.; Stark, J. Immobilized RGD Peptides on SurfaceGrafted Dextran Promote Biospecific Cell Attachment. J. Biomed. Mater. Res. 2001, 56, 390-399.

(27) Auernheimer, J.; Zukowski, D.; Dahmen, C.; Kantlehner, M.; Enderle, A.; Goodman, S. L.; Kessler, H. Titanium Implant Materials with Improved Biocompatibility through Coating with PhosphonateAnchored Cyclic RGD Peptides. ChemBioChem 2005, 6, 2034-2040.

(28) Patel, P. R.; Kiser, R. C.; Lu, Y. Y.; Fong, E.; Ho, W. C.; Tirrell, D. A.; Grubbs, R. H. Synthesis and Cell Adhesive Properties of Linear and Cyclic RGD Functionalized Polynorbornene Thin Films. Biomacromolecules 2012, 13, 2546-2553.

(29) Selhuber-Unkel, C.; López-García, M.; Kessler, H.; Spatz, J. P. Cooperativity in Adhesion Cluster Formation during Initial Cell Adhesion. Biophys. J. 2008, 95, 5424-5431.

(30) Helenius, J.; Heisenberg, C.-P.; Gaub, H. E.; Muller, D. J. SingleCell Force Spectroscopy. J. Cell Sci. 2008, 121, 1785-1791.

(31) Schmitz, J.; Benoit, M.; Gottschalk, K.-E. The Viscoelasticity of Membrane Tethers and Its Importance for Cell Adhesion. Biophys. J. 2008, 95, 1448-1459.

(32) Meister, A.; Gabi, M.; Behr, P.; Studer, P.; Vörös, J.; Niedermann, P.; Bitterli, J.; Polesel-Maris, J.; Liley, M.; Heinzelmann, H.; Zambelli, T. FluidFM: Combining Atomic Force Microscopy and Nanofluidics in a Universal Liquid Delivery System for Single Cell Applications and beyond. Nano Lett. 2009, 9, 25012507.

(33) Potthoff, E.; Guillaume-Gentil, O.; Ossola, D.; Polesel-Maris, J.; LeibundGut-Landmann, S.; Zambelli, T.; Vorholt, J. A. Rapid and Serial Quantification of Adhesion Forces of Yeast and Mammalian Cells. PLoS One 2012, 7, e52712.

(34) Potthoff, E.; Franco, D.; D’Alessandro, V.; Starck, C.; Falk, V.; Zambelli, T.; Vorholt, J. A.; Poulikakos, D.; Ferrari, A. Toward a Rational Design of Surface Textures Promoting Endothelialization. Nano Lett. 2014, 14, 1069-1079.

(35) Jaatinen, L.; Young, E.; Hyttinen, J.; Vörös, J.; Zambelli, T.; Demkó, L. Quantifying the Effect of Electric Current on Cell Adhesion Studied by Single-Cell Force Spectroscopy. Biointerphases 2016, 11, 011004. 
(36) Rowley, J. A.; Mooney, D. J. Alginate Type and RGD Density Control Myoblast Phenotype. J. Biomed. Mater. Res. 2002, 60, 217223.

(37) Grandbois, M.; Beyer, M.; Rief, M.; Clausen-Schaumann, H.; Gaub, H. E. How Strong Is a Covalent Bond? Science 1999, 283, 1727.

(38) Gomez-Casado, A.; Dam, H. H.; Yilmaz, M. D.; Florea, D.; Jonkheijm, P.; Huskens, J. Probing Multivalent Interactions in a Synthetic Host-guest Complex by Dynamic Force Spectroscopy. J. Am. Chem. Soc. 2011, 133, 10849-10857.

(39) Evans, E.; Ritchie, K. Dynamic Strength of Molecular Adhesion Bonds. Biophys. J. 1997, 72, 1541-1555.

(40) Lehenkari, P. P.; Horton, M. A. Single Integrin Molecule Adhesion Forces in Intact Cells Measured by Atomic Force Microscopy. Biochem. Biophys. Res. Commun. 1999, 259, 645-650.

(41) Sengupta, D.; Gilbert, P. M.; Johnson, K. J.; Blau, H. M.; Heilshorn, S. C. Protein-Engineered Biomaterials to Generate Human Skeletal Muscle Mimics. Adv. Healthcare Mater. 2012, 1, 785-789.

(42) Lee, J. W.; Samal, S.; Selvapalam, N.; Kim, H.-J.; Kim, K. Cucurbituril Homologues and Derivatives: New Opportunities in Supramolecular Chemistry. Acc. Chem. Res. 2003, 36, 621-630.

(43) Cohen, M.; Joester, D.; Geiger, B.; Addadi, L. Spatial and Temporal Sequence of Events in Cell Adhesion: From Molecular Recognition to Focal Adhesion Assembly. ChemBioChem 2004, 5, 1393-1399.

(44) Tian, F.; Cheng, N.; Nouvel, N.; Geng, J.; Scherman, O. A. SiteSelective Immobilization of Colloids on $\mathrm{Au}$ Substrates via a Noncovalent Supramolecular "Handcuff". Langmuir 2010, 26, 53235328.

(45) Yi, S.; Kaifer, A. E. Determination of the Purity of Cucurbit[n] uril $(\mathrm{n}=7,8)$ Host Samples. J. Org. Chem. 2011, 76, 10275-10278.

(46) Dörig, S. P. Manipulating Cells and Colloids with FluidFM; ETH Zürich: Zürich, 2013.

(47) Putman, C. A.; De Grooth, B. G.; Van Hulst, N. F.; Greve, J. A Detailed Analysis of the Optical Beam Deflection Technique for Use in Atomic Force Microscopy. J. Appl. Phys. 1992, 72, 6-12.

(48) Sader, J. E.; Chon, J. W.; Mulvaney, P. Calibration of Rectangular Atomic Force Microscope Cantilevers. Rev. Sci. Instrum. 1999, 70, 3967-3969. 